\title{
Ueber die Tetronsäuregruppe.
}

[Zweite Abhandlung. ${ }^{1}$ )

\section{Teber die Einwirkung von Acetylmandelsäurechlorid auf Natriummalonsäureester und auf Natriumcyan- essigester;}

von Richard Anschütz und Rudolf Böcker ${ }^{2}$ ).

(Eingelaufen am 13. April 1909.)

Die Uebertragung der mit dem Acetylsalicylsäurechlorid und Natriummalonsäureester durchgeführten Reaction auf das Acetylglycolsäurechlorid hatte auf einem neuen Wege in das Tetronsäuregebiet geführt. Die Reaction wurde im hiesigen chemischen Institute nach verschiedenen Richtungen hin verallgemeinert. Von dem Acetylmandelsäure- oder Acetylphenylglycolsäurechlorid aus kamen wir zu der $\alpha$-Carboxäthyl- $\gamma$-phenyltetronsäure und von dieser zu der $\gamma$-Phenyltetronsäure selbst. Wir untersuchten ferner die Umsetzung von Acetylmandelsäurechlorid mit Natriumcyanessigester und erhielten ein ölförmiges Reactionsproduct, das wir nicht zu reinigen vermochten. Allein die Analyse eines aus ihm bereiteten Silbersalzes sprach dafür, dass wir den Acetphenylglycoylcyanessigester unter Händen hatten. Die Reaction hatte offenbar einen ähnlichen Verlauf genommen, wie bei der von Anschütz und R. Fresenius aufgefundenen Bildung des Acetsalicoylcyanessigesters aus Acetylsalicylsäurechlorid und Natriumcyanessigester. ${ }^{3}$ )

1) Erste Abhandlung: Ber. d. d. chem. Ges. 36, 468 (1903).

2) Inaug.-Dissert. : Ueber Acetylmandelsäure und Acetylmandelsäurechlorid und über $\gamma$-Phenyltetronsäure von $R u d o l f$ Böcker, Bonn 1905.

3) Diese Annalen 367, 177 (1909). 
Behandelt man den Acetsalicoylcyanessigester mit Ammoniak, so geht er unter Abspaltung von Essigester und unter Bildung des Lactonringes in $\alpha$-Cyanbenzotetronsäure über:

$$
\mathrm{C}_{6} \mathrm{H}_{4}\left\{\begin{array} { l } 
{ [ 1 ] \mathrm { CN } } \\
{ [ 2 ] \mathrm { CCOCH } _ { 3 } }
\end{array} \quad \longrightarrow \quad \mathrm { C } _ { 6 } \mathrm { H } _ { 4 } \left\{\begin{array}{l}
{[1] \mathrm{C}(\mathrm{OH})=\mathrm{C} . \mathrm{CN}} \\
{[2] \mathrm{O}-\mathrm{CO}}
\end{array}\right.\right.
$$

Eine Umwandlung ganz anderer Art erleidet das ölförmige Umsetzungsproduct von Acetylmandelsäurechlorid auf Natriumcyanessigester, in dem wir geneigt sind, den Acetphenylglycoylcyanessigester zu sehen. Kocht man diese Verbindung mit Methyl- oder Aethylalkohol, oder lässt man auf sie rauchende Salzsäure bei gewöhnlicher Temperatur einwirken, so entsteht in glatter Reaction eine weisse feste Substanz. Die Analysen stimmen auf ein Condensationsproduct, das aus dem Acetphenylglycoylcyanessigester unter Aufnahme von einem Molekïl $\mathrm{W}$ asser und darauf folgender Abspaltung von einem Molekz̈l Essigsäure entstanden ist. Die neue Verbindung unterscheidet sich von der $\gamma$-Phenyl-c-carboxäthyltetronsäure durch Ersatz eines Sauerstoffatoms durch eine Imidogruppe. Es liegt also nahe, in ihr das der $\gamma$-Phenylo-carboxäthyltetronsäure entsprechende Lactam zu sehen und den Reactionsverlauf in folgender Art schematisch darzustellen:

$$
\begin{aligned}
& \mathrm{CO}_{2} \mathrm{C}_{2} \mathrm{H}_{5} \\
& \mathrm{C}_{6} \mathrm{H}_{5} \cdot \mathrm{CH} . \mathrm{CO} . \dot{\mathrm{C}} \mathrm{H} . \mathrm{CN} \\
& +\mathrm{H}_{2} \mathrm{O} \text { O.CO. } \mathrm{CH}_{3} \\
& +\underset{\mathrm{C}_{6} \mathrm{H}_{5} \cdot \mathrm{CH} \cdot \mathrm{CO} \cdot \stackrel{\mathrm{CO}_{2} \mathrm{C}_{2} \mathrm{H}_{5}}{\mathrm{CH}} \cdot \mathrm{CO} \cdot \mathrm{NH}_{2}}{\mathrm{H}_{2} \mathrm{O}} \\
& \text { O. } \mathrm{CO} . \mathrm{CH}_{3} \\
& -{ }_{\varphi} \mathrm{CH}_{3} \mathrm{CO}_{2} \mathrm{H} \quad \mathrm{CO}_{2} \mathrm{C}_{2} \mathrm{H}_{5} \quad \mathrm{CO}_{2} \mathrm{C}_{2} \mathrm{H}_{5} \\
& \mathrm{C}_{6} \mathrm{H}_{5} \text {. CH.CO. } \mathrm{CH} \quad \text { oder } \quad \mathrm{C}_{6} \mathrm{H}_{5} \cdot \mathrm{CH} \cdot \mathrm{C}(\mathrm{OH})=\mathrm{C} \\
& \dot{\mathrm{NH}}-\dot{\mathrm{CO}} \\
& \dot{\mathrm{NH}} \_\mathrm{CO}
\end{aligned}
$$

Ist diese Auffassung richtig, so haben wir in der neuen Verbindung einen Abkömmling des $\gamma$-Amid- $\beta$-oxo- 
butyrolactams oder der Tetramsäure, wie man dieses cyklische Lactam im Vergleich zur Tetronsäure nennen könnte:<smiles>CCOCC(=O)CCC(=O)OCC(=O)OCC</smiles>

Tetronsäure.

$$
\begin{aligned}
& \mathrm{CH}_{2} \cdot \mathrm{CO} \cdot \mathrm{CH}_{2} \\
& \dot{\mathrm{NH}}-\dot{\mathrm{CO}}
\end{aligned} \text { oder } \begin{aligned}
& \mathrm{CH}_{2}-\mathrm{C}(\mathrm{OH})=\mathrm{CH} \\
& \dot{\mathrm{NH}}
\end{aligned}
$$

Tetramsäure.

Während jedoch die $\gamma$-Phenyl-c-carboxäthyltetronsäure einen ausgeprägt saneren Charakter zeigt, hat die $\gamma$-Phenyl- $\alpha$-carboxäthyltetramsäure oder das $\gamma$-Phenyl$\alpha$-carboxäthyl- $\beta$-oxobutyrolactam keine saueren Eigenschaften mehr, sodass von den beiden oben gegebenen Formeln die Ketoformel mehr für sich hat.

Die Bildung des $\gamma$-Phenyl- $\alpha$-carboxäthylbutyrolactams aus dem Acetphenylglycoylcyanessigester erinnert an Lactambildungen aus in Orthostellung substituirten Benzonitrilen, bei denen ebenfalls die Umwandlung des CN in $\mathrm{CONH}_{2}$ der Lactambildung vorausgeht. Es sei auf folgende Reactionen hingewiesen:

Die Bildung von Phtalimid aus o-Cyanbenzoësäure nach Traugott Sandmeyer: $\left.:^{4}\right)$ $\mathrm{C}_{6} \mathrm{H}_{4}\left\{\begin{array}{l}{[1] \mathrm{CO}_{2} \mathrm{H}} \\ {[2] \mathrm{CN}}\end{array} \rightarrow \mathrm{C}_{6} \mathrm{H}_{4}\left\{\begin{array}{l}{[1] \mathrm{COOH}} \\ {[2] \mathrm{CONH}_{2}}\end{array} \rightarrow \mathrm{C}_{6} \mathrm{H}_{4}\left\{\begin{array}{l}{[1] \mathrm{CO}} \\ {[2] \mathrm{CO}}\end{array}>\mathrm{NH}\right.\right.\right.$

Die Bildung von Homophtalimid aus 0-Cyanbenzylcyanid bezw. 0-Cyanphenylessigsäure nach Gabriel: ${ }^{5}$ )

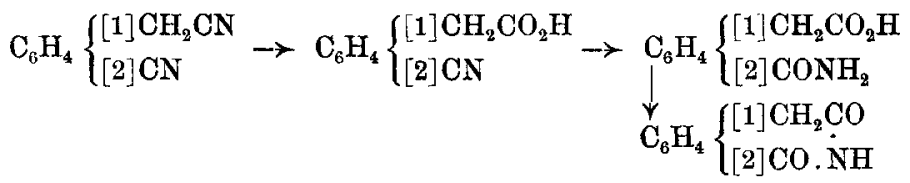

Die Bildung von $\gamma$-Cyan- $\beta$-methylisocarbostyril aus Pseudo-diacetyl-o-cyanbenzylcyanid oder $\alpha$-Cyan- $\beta$-acetoxyl- $\alpha-[2$-cyanphenyl $]$ propen nach Gabriel und

4) Ber. d. d. chem. Ges. 18, 1499 (1885).

5) Ber. d. d. chem. Ges, 20, 2502 (1887), 25, 3565 (1892). 
P o s e n e ${ }^{6}$ ) unter der Einwirkung von Alkalien:

$$
\begin{aligned}
& \begin{array}{l}
\mathrm{C}_{6} \mathrm{H}_{4}\left\{\begin{array}{l}
{[1] \mathrm{C}(\mathrm{CN})=\mathrm{C} \cdot \mathrm{CH}_{3}} \\
{[2] \mathrm{CN} \quad \text { o. } \mathrm{CO}^{2} \cdot \mathrm{CH}_{3}}
\end{array}\right. \\
\mathrm{C}_{6} \mathrm{H}_{4} \begin{cases}{[1] \mathrm{C}(\mathrm{CN})=\mathrm{C} \cdot \mathrm{CH}_{8}} \\
{[2] \mathrm{CONH}_{2}} & \text { O. } \mathrm{CO} \cdot \mathrm{CH}_{3}\end{cases}
\end{array}
\end{aligned}
$$

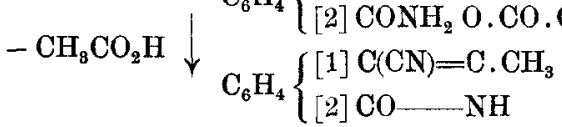

Die letztere Reaction steht der von uns beobachteten Lactambildung besonders nahe.

Ist unsere Auffassung der $\gamma$-Phenyl- $\alpha$-carboxäthyltetramsäure oder des $\gamma$-Phenyl- $\alpha$-carboxäthyl- $\beta$-oxobutyrolactams richtig, so ist diese Verbindung den eingehend untersuchten $\gamma$-Oxyisocarbostyrilen, die durch Umlagerung von Phtalimidofettsäureestern bei der Einwirkung von Natriumalkoholat entstehen, ${ }^{7}$ ) in der (onstitution ähnlich.

$$
\begin{aligned}
& \mathrm{C}_{6} \mathrm{H}_{4}\left\{\begin{array}{l}
{[1] \mathrm{CO}>\mathrm{N} . \mathrm{CH}_{2} \cdot \mathrm{CO}_{2} \mathrm{C}_{2} \mathrm{H}_{5}} \\
{[2] \mathrm{CO}}
\end{array} \begin{array}{c}
\text { Phtalyl- } \\
\text { glycocollester }
\end{array}\right. \\
& \mathrm{C}_{6} \mathrm{H}_{4}\left\{\begin{array}{l}
{[1] \cdot \mathrm{CO} \cdot \mathrm{NH}} \\
{[2] \mathrm{CO} \cdot \dot{\mathrm{CHCO}} \mathrm{C}_{2} \mathrm{H}_{5} \text { 3-caryisocarbostyril- }}
\end{array}\right. \\
& \mathrm{C}_{6} \mathrm{H}_{4}\left\{\begin{array}{l}
{[1] \mathrm{CO} \cdot \mathrm{NH}} \\
{[2] \mathrm{CO} \cdot \mathrm{CH}_{2}}
\end{array}\right. \\
& \text { oder } \\
& \mathrm{C}_{6} \mathrm{H}_{4}\left\{\begin{array}{l}
{[1] \mathrm{CO}-\mathrm{NH}} \\
{[2] \mathrm{C}(\mathrm{OH})=\dot{\mathrm{CH}}}
\end{array}\right.
\end{aligned}
$$

Die Isocarbostyrile entstehen sehr leicht aus den ihnen entsprechenden Lactonen, den Isocumarinen ${ }_{\text {, durch }}$ Einwirkung von Ammoniak. Vielleicht gelingt es auf dieselbe Weise die $\gamma$-Phenyl- $\alpha$-carboxäthyltetronsäure in das entsprechende Lactam umzuwandeln, das dann identisch sein müsste mit dem Condensationsproduct des Acetphenylglycoylcyanessigesters, wenn letzteres in der That $\gamma$-Phenyl- $\alpha$-carboxäthyl- $\beta$-oxobutyrolactam ist.

Wir haben bei dieser Betrachtung nur die Lactamformel für das Condensationsproduct als die wahrschein-

6) Ber. d. d. chem. Ges. 27, 827, 2232 (1894); 29, 2543 (1896).

ॠ) Ber. d. d. chem. Ges. 33, 980 (1900); 35, 1358, 2421, 2831 (1902); 37, 1685 (1904). 
lichste berücksichtigt, ohne indessen zu verkennen, dass auch die Imidolactonformel:

$$
\mathrm{C}_{6} \mathrm{H}_{5} \mathrm{CH}<\mathrm{O}-\dot{\mathrm{CO}}=\mathrm{CH} \cdot \mathrm{CO}_{2} \mathrm{C}_{2} \mathrm{H}_{5}
$$

nicht völlig ausgeschlossen ist.

Natürlich versuchten wir ans der $\gamma$-Phenyl- $\iota$-carboxäthyltetramsäure durch Verseifung die $\gamma$-Phenyltetram$\alpha$-carbonsäure und daraus die $\gamma$-Phenyltetramsäure selbst zu gewinnen, ohne indessen dieses Ziel bis jetzt erreichen zu können. Wir erhielten durch Einwirkung von Alkalilauge auf die $\gamma$-Phenyl-c-carboxäthyltetramsäure zwei isomere Säuren, deren Beziehung zum Ausgangsmaterial wir noch nicht mit Sicherheit festzustellen vermochten und die noch einer eingehenden Prüfung bedürfen.

\section{Ueber Acetylmandelsäure.}

Bereits 1866 versuchten A. Naquet und W. Louginin $\mathrm{e}^{8}$ ) die Acetylmandelsäure durch Kinwirkung von Acetylchlorid auf Mandelsäure zu bereiten, „um die Zweiatomigkeit dieser Säure darzuthun". Da sie jedoch das überschüssige Acetylchlorid mit Alkohol zersetzten, so erhielten sie unter dem Einflusse der entstandenen Salzsäure statt der freien Acetylmandelsäure, deren bei $73,5-74^{0}$ schmelzenden Aethylester. Allein es hat keine Schwierigkeit, auch die Acetylmandelsäure selbst auf diesem Wege au gewinnen, wenn man nur das Reactionsproduct von Acetylchlorid auf Mandelsäure nicht mit Alkohol behandelt.

Acetylmandelsäure, Schmelzp. $80^{\circ}$,

$$
\begin{gathered}
\stackrel{\mathrm{CO}_{2} \mathrm{H}}{\mathrm{C}_{6} \mathrm{H}_{5}} \dot{\mathrm{C}} \mathrm{H} . \mathrm{OCOCH}_{3},
\end{gathered}
$$

erhält man als krystallinische Masse, wenn man trockne Mandelsäure mit etwas mehr als der berechneten Menge Acetylchlorid gelinde auf dem Wasserbade erwärmt bis sich die berechnete Menge Salzsäure entwickelt hat.

8) Compt. rend. 62, 430 (1866); diese Annalen 139, 299 (1866). 
Nach dem Erkalten erstarrt der syrupöse Rückstand krystallinisch. Man löst zweckmässig das Rohproduct in wenig Chloroform and fällt mit Petroläther unter Umrühren die Acetylmandelsäure als weisses, lockeres Pulver aus. Die Acetylmandelsäure ist leicht löslich in Alkohol, Aether, Chloroform und Benzol, schwer löslich in kaltem, leichter löslich in heissem Wasser, aus dem sie beim Erkalten in feinen Nadeln krystallisirt, die an der Luft, schneller im Exsiccator verwittern und $z u$ einem weissen Pulver zerfallen. Die so von Krystallwasser befreite Säure schmilzt wie die aus Chloroformlösung mit Petroläther abgeschiedene Säure bei $80^{\circ}$. Die aus Wasser umkrystallisirte Säure wurde abgesangt, zwischen Fliesspapier abgepresst und dann zu den beiden folgenden Krystallwasser-Bestimmungen verwendet, aus denen folgt, dass sie 1 Mol. Krystallwasser enthält.

I. $0,8550 \mathrm{~g}$ verloren bei $60-70^{\circ}$, unter stark vermindertem Druck bis zur Gewichtsconstanz getrocknet, $0,0745 \mathrm{~g}$.

II. $1,0745 \mathrm{~g}$ verloren $0,0895 \mathrm{H}_{2} \mathrm{O}$.

\begin{tabular}{cccc} 
& Ber. für. & \multicolumn{2}{c}{ Gef. } \\
& $\mathrm{C}_{10} \mathrm{H}_{10} \mathrm{O}_{4} \cdot \mathrm{H}_{2} \mathrm{O}$ & I & II \\
$\mathrm{H}_{2} \mathrm{O}$ & 8,49 & 8,71 & 8,33
\end{tabular}

Auch unter stark vermindertem Druck lässt sich die von Krystallwasser befreite Acetylmandelsäure nicht unzersetzt destilliren. Lässt man die aus Wasser krystallisirte Säure mit rauchender Salzsäure übergossen in einem lose verschlossenen Gefäss längere Zeit stehen, so geht sie allmählich in Lösung. Beim Abdunsten der Lösung im Exsiccator über Aetzkali und Schwefelsäure blieb Mandelsäure zurück. Auch durch Kochen der Acetylmandelsänre mit Alkoholen geht sie unter Bildung von Fssigsäureester in Mandelsäure ïber.

Die Hydrolyse der auch gegen kochendes Wasser recht beständigen Acetylmandelsäure untersuchte auf Veranlassung des Einen von uns (A.) Herr Julius Rath im Rahmen einer Arbeit, die einen Vergleich der Hydro- 
lyse acetylirter Oxysäuren bezweckte und auf deren Ergebnisse hier verwiesen werden soll. ${ }^{9}$ )

Die Analysen I und II sind mit wasserfreier Acetylmandelsänre ausgeführt, die aus Chloroformlösung mit Petroläther abgeschieden worden war. Zu Analyse III diente ein Präparat, das aus Krystallwasser haltiger, durch Einwirkung von Wasser auf Acetylmandelsäurechlorid bereiteter AcetylmandeIsäure unter vorsichtigem Erwärmen im Vacuum dargestellt wurde.

I. $0,1060 \mathrm{~g}$ gaben $0,2385 \mathrm{CO}_{2}$ und $0,0505 \mathrm{H}_{2} \mathrm{O}$.

II. $0,1775 \mathrm{~g} \quad, 0,3940 \mathrm{CO}_{2} \quad " 0,0840 \mathrm{H}_{2} \mathrm{O}$.

III. $0,1555 \mathrm{~g} \quad " \quad 0,3510 \mathrm{CO}_{\mathrm{z}}, \quad 0,0770 \mathrm{H}_{2} \mathrm{O}$.

$\begin{array}{ccccc} & \text { Ber. für } & & \text { Gef. } & \\ & \mathrm{C}_{10} \mathrm{H}_{10} \mathrm{O}_{4} & \text { I } & \text { II } & \text { III } \\ \mathrm{C} & 61,86 & 61,36 & 61,23 & 61,56 \\ \mathrm{H} & 5,15 & 5,45 & 5,35 & 5,34 \\ \mathrm{O} & 32,99 & - & - & -\end{array}$

Das Ammoniumsalz, entsteht durch Einleiten von trocknem Ammoniakgas in die Chloroformlösung der Acetylmandelsäure. Der sich sofort abscheidende Niederschlag krystallisirt aus seiner alkoholischen Lösung nach Versetzen mit Chloroform und Petroläther in glänzenden weissen Schuppen, die sich leicht in Wasser lösen.

I. $0,1449 \mathrm{~g}$ gaben $0,3010 \mathrm{CO}_{2}$ und $0,0843 \mathrm{H}_{2} \mathrm{O}$.

II. $0,3220 \mathrm{~g} \Rightarrow 18,5 \mathrm{ccm}$ Stickgas bei $21,5^{\circ}$ u. $765 \mathrm{~mm}$ Druck.

\begin{tabular}{cccc} 
& Ber. für & \multicolumn{2}{c}{ Gef. } \\
& $\mathrm{C}_{10} \mathrm{H}_{18} \mathrm{NO}_{4}$ & I & II \\
$\mathrm{C}$ & 56,84 & 56,65 & - \\
$\mathrm{H}$ & 6,20 & 6,52 & - \\
$\mathrm{N}$ & 6,65 & - & 6,57 \\
$\mathrm{O}$ & 30,31 & - & -
\end{tabular}

Acetylmandelsäurechlorid, Siedep. ${ }_{12} 132^{\circ}$ (Badtempe$\left.\operatorname{ratur} 150^{\circ}\right)$,

$$
\begin{aligned}
& \mathrm{COCl} \\
& \mathrm{C}_{6} \mathrm{H}_{5} \dot{\mathrm{CHOCOCH}} \\
& \hline
\end{aligned}
$$

wird dargestellt, indem man $30 \mathrm{~g}$ vorher geschmolzene und wieder erstarrte Acetylmandelsäure mit etwas mehr

9) Diese Annalen 358, 114 (1907). 
als der äquimolekularen Menge (34 statt 32 g) Phosphorpentachlorid zusammenbringt unter Kühlung des Reactionsgefässes mit kaltem Wasser. Nach der ersten Einwirkung verläuft die Reaction ruhig unter lebhafter Entwickelung von Salzsäure, durch Eintauchen des Gefässes in warmes Wasser wird sie beendigt. Bei der Destillation unter vermindertem Druck geht das Acetylmandelsäurechlorid als wasserhelle Flüssigkeit über unter Hinterlassung eines schwarzen Rückstandes, dabei wurden unter verschiedenen Druckverhältnissen folgende Siedepunkte beobachtet:

\begin{tabular}{|c|c|c|}
\hline Siedep. & Druck & Badtemperatur \\
\hline $129^{\circ}$ & $10 \mathrm{~mm}$ & $138-140^{\circ}$ \\
\hline $131^{\circ}$ & $11 \mathrm{~mm}$ & \multirow{2}{*}{$145-150^{\circ}$} \\
\hline $132^{\circ}$ & $12 \mathrm{~mm}$ & \\
\hline $138^{\circ}$ & $15 \mathrm{~mm}$ & \multirow{3}{*}{$160-160^{\circ}$} \\
\hline $140^{\circ}$ & $16 \mathrm{~mm}$ & \\
\hline $142^{\circ}$ & $18 \mathrm{~mm}$ & \\
\hline
\end{tabular}

Die Ausbeute an reinem Chlorid bleibt beträchtlich hinter der berechneten zurïck. Bei der Destillation des Rohproductes wird ein um so grösserer Theil des Chlorides zersetzt je mehr Acetylmandelsäure man verwendet hat. Aus $20 \mathrm{~g}$ Acetylmandelsäure wurden z. B. $16 \mathrm{~g}$, also $73 \mathrm{pC}$., aus $90 \mathrm{~g}: 64 \mathrm{~g}$, also $65 \mathrm{pC}$. der berechneten Menge Chlorid gewonnen.

Das Acetylmandelsäurechlorid ist eine wasserhelle. ölige Flüssigkeit, die an der Luft schwach raucht, indem sie zugleich nach Salzsäure und an Bittermandelöl erinnernd riecht. In Chloroform, Aether, Ligroïn und Benzol löst sich das Chlorid leicht auf.

I. $0,1776 \mathrm{~g}$ gaben $0,3627 \mathrm{CO}_{2}$ und $0,0695 \mathrm{H}_{2} \mathrm{O}$.

II. $0,2015 \mathrm{~g} \# \quad 0,4073 \mathrm{CO}_{2} n \quad 0,0780 \mathrm{H}_{2} \mathrm{O}$.

III. $0,2505 \mathrm{~g}, " 0,1705 \mathrm{AgCl}$.

\begin{tabular}{lcccc} 
& Ber. für & \multicolumn{3}{c}{ Gef. } \\
& $\mathrm{C}_{10} \mathrm{H}_{9} \mathrm{O}_{3} \mathrm{Cl}$ & $\mathrm{I}$ & II & III \\
$\mathrm{C}$ & 56,47 & 55,70 & 55,13 & - \\
$\mathrm{H}$ & 4,27 & 4,38 & 4,33 & - \\
$\mathrm{Cl}$ & 16,68 & - & - & 16,83 \\
$\mathrm{O}$ & 22,58 & - & - & -
\end{tabular}


Mit Wasser setzt sich das Acetylmandelsäurechlorid allmählich in Acetylmandelsäure, mit Alkohol in den von Naquet und Louginine zuerst erhaltenen Acetymandelsäureäthylester um. Lässt man das Chlorid mit überschüssigem Alkohol einige Tage in einem verschlossenen Gefäss stehen, so tritt beim Oeffnen des Gefässes starker Geruch nach Essigester anf, ein Beweis, dass sich unter diesen Umständen das Acetyl als Essigester abgespalten hat.

Bei Abkühlung mittelst flüssiger Luft konnte das Chlorid wohl zum Erstarren, aber nicht 'zur Krystallisation gebracht werden.

Acetylmandelsäureamid, Schmelzp.,112-113\%,

$$
\begin{gathered}
\text { CONH }_{2} \\
\mathrm{C}_{6} \mathrm{H}_{5} \dot{\mathrm{CHO}} . \mathrm{CO} . \mathrm{CH}_{3}
\end{gathered},
$$

fällt gemischt mit Salmiak unter starker Erwärmung aus, wenn man trocknes Ammoniakgas in eine ziemlich concentrirte ätherische Lösung des Chlorides einleitet. In warmem Wasser ist das Amid leicht löslich und krystallisirt beim Erkalten in feinen, weissen, büschelförmig angeordneten Nadeln.

$\begin{array}{ccc}0,1305 \text { g gaben } 0,2970 \mathrm{CO}_{2} & \text { und } & 0,0725 \mathrm{H}_{2} \mathrm{O} . \\ & \text { Ber. für } \mathrm{C}_{10} \mathrm{H}_{11} \mathrm{NO}_{3} & \text { Gef. } \\ \mathrm{C} & 62,14 & 62,08 \\ \mathrm{H} & 5,74 & 6,21 \\ \mathrm{~N} & 7,27 & - \\ \mathrm{O} & 24,85 & -\end{array}$

Acetylmandelsäureanilid, Schmelzp. 117,50,

$$
\begin{gathered}
\mathrm{CONHC}_{6} \mathrm{H}_{5} \\
\mathrm{C}_{6} \mathrm{H}_{5} \dot{\mathrm{CHO}} . \mathrm{CO} . \mathrm{CH}_{8}
\end{gathered},
$$

aus der ätherischen Lösung des Chlorides durch Zasatz der dimolekularen Menge Anilin gefällt, mit Wasser von Anilinchlorhydrat befreit, bildet aus verdünntem Alkohol umkrystallisirt feine weisse Nadeln.

I. $0,1908 \mathrm{~g}$ gaben $0,4974 \mathrm{CO}_{2}$ und $0,1000 \mathrm{H}_{2} \mathrm{O}$.

II. $0,1155 \mathrm{~g} \quad, \quad 0,3015 \mathrm{CO}_{2}, 0,0645 \mathrm{H}_{2} \mathrm{O}$. 


\begin{tabular}{cccc} 
& Ber. für & \multicolumn{2}{c}{ Gef. } \\
& $\mathrm{C}_{\mathbf{1} 6} \mathrm{H}_{15} \mathrm{NO}_{3}$ & $\mathrm{I}$ & II \\
$\mathrm{C}$ & 71,33 & 71,10 & 71,19 \\
$\mathrm{H}$ & 5,62 & 5,83 & 5,61 \\
$\mathbf{N}$ & 5,22 & - & - \\
$\mathrm{O}$ & 17,83 & - & -
\end{tabular}

Acetylmandelsäure-p-phenetidid, Schmelzp. $157^{\circ}$.

$$
\begin{gathered}
\text { CONH }[4] \mathrm{C}_{6} \mathrm{H}_{4} \mathrm{OC}_{2} \mathrm{H}_{5} \\
\mathrm{O}_{6} \mathrm{H}_{5} \dot{\mathrm{CHO}} \mathrm{CO} . \mathrm{CH}_{3}
\end{gathered}
$$

wird wie das Anilid dargestellt, krystallisirt in feinen weissen Nädelchen und löst sich in warmem Ligroïn.

I. $0,1740 \mathrm{~g}$ gaben $0,4405 \mathrm{CO}_{2}$ und $0,0965 \mathrm{H}_{2} \mathrm{O}$.

II. $0,4515 \mathrm{~g}, \quad 19 \mathrm{ccm}$ Stickgas bei $23^{\circ}$ u. 766,5 mm Druck. Bè̀r. fürr $\mathrm{C}_{18} \mathrm{H}_{18} \mathrm{NO}_{4}$

C 68,97

$\mathrm{H}$

6,11

4,48

Gef.

$\mathbf{N}$

20,44

$\begin{array}{cc}\text { I } & \text { II } \\ 69,04 & - \\ 6,16 & - \\ - & 4,77 \\ - & -\end{array}$

Acetylmandelsäurepiperidid, Schmelzp. 98

$$
\begin{gathered}
\mathrm{CONHC}_{5} \mathrm{H}_{10} \\
\mathrm{C}_{6} \mathrm{H}_{5} \mathrm{CHO} . \mathrm{COCH}_{3}
\end{gathered}
$$

wird wie das Anilid dargestellt, krystallisirt aus Alkohol in glänzenden, kleinen, weissen Nadeln und löst sich in warmem Ligroïn.

I. $0,1072 \mathrm{~g}$ gaben $0,2695 \mathrm{CO}_{2}$ und $0,0750 \mathrm{H}_{2} \mathrm{O}$.

II. $0,1147 \mathrm{~g}, 0,2895 \mathrm{CO}_{2} \# 0,0792 \mathrm{H}_{2} \mathrm{O}$.

III. $0,5040 \mathrm{~g} \quad, \quad 23,4 \mathrm{ccm}$ Stickgas bei $12^{\circ}$ u. $772 \mathrm{~mm}$ Druck. Ber. für. Gef.

$\begin{array}{ccccc} & \mathrm{C}_{15} \mathrm{H}_{18} \mathrm{NO}_{3} & \text { I } & \text { II } & \text { III } \\ \mathrm{C} & 68,92 & 68,57 & 68,84 & - \\ \mathrm{H} & 7,33 & 7,83 & 7,72 & - \\ \mathrm{N} & 5,37 & - & - & 5,22 \\ \mathrm{O} & 18,38 & - & - & -\end{array}$

2. Acetylmandelsäurechlorid u.Natriummalonsäureäthylester. $\gamma$-Phenyl- $\alpha$-carboxäthyltetronsäure, Schmelzp. $140^{\circ}$, $\mathrm{C}_{6} \mathrm{H}_{5} \mathrm{CH}<\mathrm{O}-\mathrm{C}(\mathrm{OH})=\mathrm{C} . \mathrm{CO}_{2} \mathrm{C}_{2} \mathrm{H}_{5}$

Behandelt man Acetylmandelsäurechlorid mit Natriummalonsäureäthylester, so gelingt es ebenso wenig, wie 
bei dem Acetylsalicylsäurechlorid, die Reaction bei dem ersten Product der Umsetzung festzuhalten, sondern unter Abspaltung von Acetyl and Aethoxyl als Essigester entsteht das Lacton: die $\gamma$-Phenyl- $\alpha$-carboxäthyltetronsäure. Man presst $8,2 \mathrm{~g}$ Natrium in Form von feinem Draht in $250 \mathrm{ccm}$ trocknes Benzol, fügt $56,4 \mathrm{~g}$ Malonsäureäthylester hinzu und versetzt, sobald der Natriummalonsäureester fertig gebildet ist, mit $25 \mathrm{~g}$ Acetylmandelsäurechlorid: auf $3 \mathrm{Mol}$. Natriummalonsäureäthylester verwendet man 1 Mol. Chlorid. Unter Erwärmung tritt die Reaction ein, das Gemisch färbt sich erst gelb, dann braun und ein missfarbiger Niederschlag setzt sich ab. Nach 6-7 stündigem Erhitzen auf dem Wasserbade und häufigem Umschütteln wurde der Niederschlag abfiltrirt und in warmem Wasser gelöst. Aus dieser Lösung scheidet conc. Salzsäure die $\gamma$-Phenyl- $\alpha$-carboxäthyltetronsäure als gelblich weissen Niederschlag $a b$, der in verdünntem heissem Alkohol gelöst beim Erkalten in weissen Nädelchen krystallisirt.

I. $0,1126 \mathrm{~g}$ gaben $0,2590 \mathrm{CO}_{2}$ und $0,0515 \mathrm{H}_{2} \mathrm{O}$.

II. $0,1095 \mathrm{~g} \quad, \quad 0,2520 \quad \mathrm{CO}_{2} \quad \# \quad 0,0515 \mathrm{H}_{2} \mathrm{O}$.

III. $0,1130 \mathrm{~g} \quad, \quad 0,2595 \mathrm{CO}_{2} \quad, \quad 0,0520 \mathrm{H}_{2} \mathrm{O}$.

\begin{tabular}{lcccc} 
& Ber. für & \multicolumn{3}{c}{ Gef. } \\
& $\mathrm{C}_{13} \mathrm{H}_{18} \mathrm{O}_{5}$ & I & II & III \\
$\mathrm{C}$ & 62,88 & 62,73 & 62,77 & 62,63 \\
$\mathrm{H}$ & 4,87 & 5,12 & 5,26 & 5,15 \\
$\mathrm{O}$ & 32,25 & - & - & -
\end{tabular}

Die $\gamma$-Phenyl- $\alpha$-carboxäthyltetronsäure ist eine Säure, die Kohlensäure und salpetrige Säure aus ihren Salzen austreibt; ihre wässrige Lösung reagirt sauer und vermag die Salze einiger Schwermetalle unmittelbar za fällen. Andere Salze erhält man durch Fällen der wässrigen Lösung des $\gamma$-phenyl- $\alpha$-carboxäthyltetronsauren Natriums mit Metallsalzlösungen als schwerlösliche Niederschläge.

Das Ammoniumsalz entsteht beim Einleiten von trocknem Ammoniakgas in die Benzollösung der $\gamma$-Phenyl$\alpha$-carboxäthyltetronsäure als zuerst gallertartige, dann 
pulverig werdende weisse Ausscheidung; aus ätherischer Lösung wird das Ammoniumsalz sofort pulverig gefällt. Diese Reaction spricht für die Enolformel der Säure, die übrigens auch durch die saure Reaction und durch die Fähigkeit der Säure, Carbonate zu zerlegen, befürwortet wird.

Das Natriumsalz entsteht einmal bei der Einwirknng von Acetylmandelsäurechlorid auf einen Ueberschuss von Natriummalonsäureäthylester, dann beim Vermischen einer alkoholischen Natriumäthylatlösung mit der berechneten Menge $\gamma$-Phenyl- $\alpha$-carboxäthyltetronsäure. Das Natriumsalz ist sowohl in Wasser, als in Alkohol sehr leicht löslich und wird daher mit Aether aus der alkoholischen Lösung abgeschieden:

$$
\begin{array}{rcr}
0,1405 \mathrm{~g} \text { gaben } 0,0360 \mathrm{SO}_{4} \mathrm{Na}_{2} . & \\
& \text { Ber. für } \mathrm{C}_{13} \mathrm{H}_{11} \mathrm{NaO}_{5} & \text { Gef. } \\
\mathrm{Na} & 8,53 & 8,31
\end{array}
$$

Das Ferrisalz, $\left(\mathrm{C}_{13} \mathrm{H}_{11} \mathrm{O}_{5}\right)_{3} \mathrm{Fe}$, fällt beim Versetzen einer warmen wässrigen Lösung der $\gamma$-Phenyl-c $c$-carboxäthyltetronsäure mit Eisenchloridlösung als dicker, hellrother Niederschlag auch schon aus ziemlich verdünnter Lösung aus. Das abfiltrirte Salz wurde erst über Phosphorpentoxyd, dann bei $95^{\circ}$ bis zur Gewichtsconstanz getrocknet und analysirt.

$0,2305 \mathrm{~g}$ gaben $0,0220 \mathrm{Fe}_{2} \mathrm{O}_{3}$.

$$
\begin{array}{ccc} 
& \text { Ber. für } \mathrm{C}_{39} \mathrm{H}_{93} \mathrm{O}_{15} \mathrm{Fe} & \text { Gef. } \\
\mathrm{Fe} & 7,02 & 6,68
\end{array}
$$

Das Ferrisalz ist sehr leicht in Alkohol und Chloroform mit dunkelrother Farbe löslich; auch löst es sich in viel überschüssigem Eisenchlorid wieder anf.

Das Kobaltsalz, $\left(\mathrm{C}_{13} \mathrm{H}_{11} \mathrm{O}_{5}\right)_{2} \mathrm{Co}$, scheidet sich auf $\mathrm{Zu}-$ satz einer Kobaltsalzlösung zu der Lösung des Ammoniumoder Natriumsalzes nach einiger Zeit in glänzenden, dunkelrothen Kryställchen aus, die schon an der Luft, schneller im Exsiccator zu einem hellrosarothen Pulver zerfallen. Die Analyse des bei $110^{\circ}$ getrockneten Salzes zeigte, dass das Salz wasserfrei geworden war. 
$0,1347 \mathrm{~g}$ gaben 0,0144 Co.

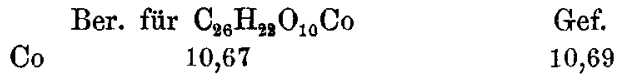

Anf ähnliche Weise wurden erhalten, aber nicht analysirt, das Nickelsalz, als hellgrünes krystallinisches Pulver, das Bleisalz weiss krystallinisch, das Magnesiumsalz weisskörnig, das Kupfersalz hellblaugrün, mit grüner Farbe in Chloroform löslich.

\section{Spaltung der $\gamma$-Phenyl- $\alpha$-carboxäthyltetronsäure,}

$\gamma$-Phenyltetronsäure, Schmelzp. 127,5-128,50,

$$
\mathrm{C}_{8} \mathrm{H}_{5} \mathrm{CH}<\underset{\mathrm{O}-\mathrm{C}(\mathrm{OH})=\mathrm{CH}}{\mathrm{CO}},
$$

wird aus $\gamma$-Phenyl-ce-carboxäthyltetronsäure durch Erhitzen mit Kalilauge in Form ihres Kaliumsalzes erhalten. $10 \mathrm{~g}$ der $\gamma$-Phenyl-c-carboxäthyltetronsäure werden mit einer Lösung von 7,5 g Kaliumhydroxyd in $50 \mathrm{ccm}$ Wasser 7-8 Stunden lang unter Rückflusskühlung gekocht. Nach dem Frkalten filtrirt man durch ein Asbestfilter, wäscht mit etwas Wasser nach und fällt die $\gamma$-Phenyltetronsäure mit Salzsäure oder verdünnter Schwefelsäure aus. Um sie zu reinigen, führt man sie durch Einleiten von trocknem Ammoniakgas in ihre alkoholische, mit etwas Ligroïn versetzte Lösung, in Ammoniumsalz über. Die aus diesem abgeschiedene Säure wird in Natriumsalz umgewandelt, daraus von neuem gefällt und aus heissem Benzol umkrystallisirt. Sie löst sich leicht in Alkohol, schwerer in heissem Wasser, Chloroform und Benzol, schwer in Aether und überhaupt nicht in Ligroïn. Aus Wasser krystallisirt die $\gamma$-Phenyltetronsäure in weissen, weichen, verfilzten Nädelchen, die sich federförmig zusammenordnen. Zur Elementaranalyse muss man die bei gewöhnlicher Temperatur im Vacuumexsiccator getrocknete Sänre verwenden. Versucht man die Säure unter vermindertem Druck bei $95-100^{\circ} \mathrm{zu}$ trocknen, so nimmt sie allmählich an Gewicht ab und die Analysen zeigten, dass der Kohlenstoff- 
gehalt gestiegen ist, während der Wasserstoffgehalt abgenommen hat. Nach den von L. Wolff ${ }^{10}$ ) bei der Umwandlung der Tetronsäure in die ,Anhydrotetronsäure" gemachten Beobachtungen wird man wohl auch von der $\gamma$-Phenyltetronsäure eine Anhydroverbindung erhalten können, wir haben sie jedoch in dieser Richtung noch nicht weiter untersucht. Die im Vacuumexsiccator bei gewöhnlicher Temperatur getrocknete $\gamma$-Phenyltetronsäure gab bei der Elementaranalyse gut auf die Formel $\mathrm{C}_{10} \mathrm{H}_{8} \mathrm{O}_{3}$ stimmende Werthe.

I. $0,1570 \mathrm{~g}$ gaben $0,3910 \mathrm{CO}_{2}$ und $0,0638 \mathrm{H}_{2} \mathrm{O}$.

II. $0,1408 \mathrm{~g} \quad " \quad 0,3525 \quad \mathrm{CO}_{2} \quad, \quad 0,0562 \mathrm{H}_{2} \mathrm{O}$.

III. $0,1230 \mathrm{~g} \quad, \quad 0,3065 \quad \mathrm{CO}_{2} \quad, \quad 0,0510 \quad \mathrm{H}_{2} \mathrm{O}$.

\begin{tabular}{lcccc} 
& Ber. für & \multicolumn{3}{c}{ Gef. } \\
& $\mathrm{C}_{\mathbf{1 0}} \mathrm{H}_{8} \mathrm{O}_{3}$ & I & II & III \\
$\mathrm{C}$ & 68,16 & 67,92 & 68,28 & 67,96 \\
$\mathrm{H}$ & $\mathbf{4 , 5 8}$ & 4,55 & $\mathbf{4 , 4 6}$ & 4,64 \\
$\mathrm{O}$ & 27,26 & - & - & -
\end{tabular}

Eine Titration der Säure hatte folgendes Ergebnis:

$0,2690 \mathrm{~g}$ brauchten $15,25 \mathrm{ccm} \mathrm{n} / 10$-Natronlauge (Factor 0,109).

$\begin{array}{ccc} & \text { Ber. für } \mathrm{C}_{10} \mathrm{H}_{8} \mathrm{O}_{3} & \text { Gef. } \\ \mathrm{NaOH} & \mathbf{2 2 , 7 3} & \mathbf{2 2 , 4 7}\end{array}$

Wie die Tetronsäure giebt die $\gamma$-Phenyltetronsäure mit Eisenchlorid eine tief dunkelrothe Färbung.

Das Ammoniumsalz,<smiles>O=C(CNC(=O)c1ccccc1)OCc1ccccc1</smiles>

schmilzt bei $148-149^{\circ}$ unter Zersetzung und wird durch Einleiten von trocknem Ammoniak in die mit etwas Alkohol versetzte Lösung der Säure in Benzol dargestellt. Das abgeschiedene Salz wird in warmem Alkohol gelöst und durch Versetzen mit Aether beim Erkalten zur Krystallisation gebracht. Es bildet fast weisse, sternförmig angeordnete Schuppen.

I. $0,1195 \mathrm{~g}$ gaben $0,2725 \mathrm{CO}_{2}$ und $0,0670 \mathrm{H}_{2} \mathrm{O}$.

II. $0,1719 \mathrm{~g} \quad, \quad 0,3912 \quad \mathrm{CO}_{2} \quad, \quad 0,0945 \mathrm{H}_{2} \mathrm{O}$.

10) Diese Annalen 291, 251 (1896). 


\begin{tabular}{lccc} 
& Ber. für. & \multicolumn{2}{c}{ Gef. } \\
& $\mathrm{C}_{10} \mathrm{H}_{11} \mathrm{O}_{3} \mathrm{~N}$ & I & II \\
$\mathrm{C}$ & 62,14 & 62,19 & 62,07 \\
$\mathrm{H}$ & 5,74 & 6,26 & $\mathbf{6 , 1 5}$ \\
$\mathrm{N}$ & $\mathbf{7 , 2 7}$ & - & - \\
$\mathrm{O}$ & $\mathbf{2 4 , 8 5}$ & - & -
\end{tabular}

Das Natriumsalz schmilzt zwischen 105 und $110^{\circ} \mathrm{zu}$ einer klaren, gelblichen Flüssigkeit, die beim Erkalten glasartig erstarrt. Man bereitet es in reinem Zustand durch Behandeln der Säure mit der berechneten Menge Natriumäthylat in alkoholischer Lösung.

I. $0,1115 \mathrm{~g}$ gaben $0,0392 \mathrm{SO}_{4} \mathrm{Na}_{2}$.

II. $0,1880 \mathrm{~g} \quad, \quad 0,0672 \mathrm{SO}_{4} \mathrm{Na}_{2}$.
Ber. für
$\begin{array}{cccc} & \mathrm{C}_{10} \mathrm{H}_{7} \mathrm{O}_{3} \mathrm{Na} & \mathrm{I} & \text { II } \\ \mathrm{Na} & 11,64 & 11,40 & 11,59\end{array}$
Gef.

$\gamma$-Phenyl-oximidotetronsäure, $\gamma$-Phenyl- $\beta$-oxo- $\alpha$-oximidobutyrolacton,<smiles>O=C(O)C1OC(=O)C(c2ccccc2)OC1=O</smiles>

schmilzt bei $92-93^{\circ}$ unter Aufschäumen und zersetzt sich bei weiterem Frhitzen vollständig. Die $\gamma$-Phenyltetronsäure zeigt die von L. Wolff beschriebene Nitrosoreaction in aller Schärfe. Versetzt man die verdünnte Lösung eines tetronsauren Alkalisalzes mit Natriumnitrit und säuert vorsichtig mit Essigsäure an, so tritt eine violette Färbung auf, die beim Erwärmen verschwindet und durch weiteren Zusatz der Säure in gelb umschlägt. Zur Darstellung der $\gamma$-Phenyloximidotetronsäure versetzt man die mit wenig Wasser vermischte $\gamma$-Phenyltetronsäure mit der conc. Lösung der berechneten Menge Natriumnitrit und lässt einige Zeit stehen. Es bildet sich sofort eine dunkelviolette gefärbte Lösung des Natriumsalzes der $\gamma$-Phenyloximidotetronsäure. Säuert man diese Lösung unter Fiskühlung mit 12procentiger Salzsäure an, so fällt der grösste Theil der Säure in hellgelben Blättchen aus. Die in Wasser und Alkohol leicht lösliche Säure zersetzt sich äusserst leicht schon beim Erwärmen der wässrigen Lösung. 
$0,1270 \mathrm{~g}$ gaben $0,2695 \mathrm{CO}_{2}$ und $0,0425 \mathrm{H}_{2} \mathrm{O}$.

$\begin{array}{ccc} & \text { Ber. für } \mathrm{C}_{10} \mathrm{H}_{7} \mathrm{O}_{4} \mathrm{~N} & \text { Gef. } \\ \mathrm{C} & 58,51 & 57,87 \\ \mathrm{H} & 3,44 & 3,74 \\ \mathrm{~N} & 6,84 & - \\ \mathrm{O} & 31,21 & -\end{array}$

Die $\gamma$-Phenyl- $\iota$-carboxäthyl-tetronsäure giebt keine Isonitrosoverbindung, vielmehr wirkt sie auf das Natriumnitrit bei gelindem Erwärmen unter Entwickelung rother Dämpfe ein. Die Stelle, an die die Isonitrosogruppe treten sollte, ist durch Carboxäthyl besetzt. Die $\gamma$-Phenyl- $\alpha$-isonitroso-tetronsäure enthält die Oximido- oder Isonitrosogruppe am $\ell$-Kohlenstoffatom, so dass die Verbindung kein Abkömmling der $\gamma$-Phenyltetronsäure mehr ist, sondern auf das $\gamma$-Phenyl- $\beta$-oxobutyrolacton zurückgeführt werden muss. Daher haben wir sie auch entsprechend der oben für sie aufgestellten Structurformel als $\gamma$-Phenyl$\beta$-oxo- $\alpha$-oximidobutyrolacton bezeichnet.

In einem nicht luftdicht verschlossenen Gefässe zersetzt sich das $\gamma$-Phenyl- $\beta$-oxo- $\alpha$-oximidobutyrolacton allmählich, indem es sich entfärbt und sein Schmelzpunkt steigt.

\section{Acetylmandelsäurechlorid und Natriumcyanessigester.}

Bei der Einwirkung dieser beiden Verbindungen auf einander entsteht offenbar der Phenylacetoxyacetylcyanessigester oder Acetphenylglycoylcyanessigester:

$$
\begin{aligned}
& \text { CN }
\end{aligned}
$$

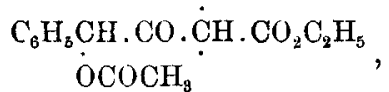

wie bei der Einwirkung von Acetylsalicylsäurechlorid auf Natriumcyanessigester der Acetsalicoylcyanessigester,

$$
\mathrm{C}_{6} \mathrm{H}_{4}\left\{\begin{array}{l}
{[1] \mathrm{CO} \cdot \dot{\mathrm{CH}} \cdot \mathrm{CO}_{2} \mathrm{C}_{2} \mathrm{H}_{5},} \\
{[2] \mathrm{OCOCH}}
\end{array}\right.
$$

erhalten wurde. Allein bei der ölförmigen Beschaffenheit dieses nicht unzersetzt destillirbaren Productes gelang es uns nicht, den Acetphenylglycoylcyanessigester in. 
reinem Zustande zu gewinnen, wohl aber lieb sich sein Vorhandensein durch die Analyse seines Silbersalzes wahrscheinlich machen.

Presst man in eine ätherische Lösung von $40 \mathrm{~g}$ Cyanessigester 8,2 $\mathrm{g}$ feinen Natriumdraht unter Kühlung und fügt, nachdem der Natriumcyanessigester sich gebildet hat, $25 \mathrm{~g}$ Acetylmandelsäurechlorid hinzu, so wirken beide Verbindungen unter starker Wärmeentwickelung auf einander ein. Das Gemisch wird zur Vollendung der Umsetzung etwa 16 Stunden lang am Rückflusskühler unter häufigem Umschütteln bis zum Sieden des Aethers erhitzt, wobei sich die Masse schwach gelb färbt. Nach Beendigung der Reaction wird der Niederschlag abgesaugt, in warmem Wasser gelöst und die Lösung mit Salzsäure angesäuert. Es scheidet sich ein gelbbraunes Oel aus, das sich im Verlauf eines Tages am Boden des Gefässes sammelt. Die darüber stehende Flüssigkeit wird abgegossen, das Oel in Aether aufgenommen und die ätherische Lösung mit Chlorcalcium getrocknet. Nach dem Abdestilliren des Aethers konnte das trockne Oel anch durch starkes Abkühlen nicht zur Krystallisation gebracht werden. Das bräunlichgelbe Oel wurde nicht analysirt, es zeigte saure Eigenschaften, löst sich leicht in heißem Wasser mit saurer Reaction und zersetzt Sodalösung unter Kohlensäureentwickelung. Die wässrige Lösung des ölförmigen Esters oder die seines Natriumsalzes giebt mit den Salzlösungen der Schwermetalle ganz ähnliche Fällungen wie die der $\gamma$-Phenyl- $\boldsymbol{c}$-carboxäthyltetronsänre, nur weniger lebhaft gefärbt.

Das Sillersalz,

$$
\mathrm{C}_{6} \mathrm{H}_{5} \mathrm{CH}<\underset{\mathrm{OCO} . \mathrm{CH}_{3} \mathrm{CN}}{\mathrm{C}(\mathrm{CAg})=\underset{\mathrm{C}}{\mathrm{C}} \mathrm{CO}_{2} \mathrm{C}_{2} \mathrm{H}_{5}},
$$

wird durch Fällung des leicht in Wasser löslichen Ammoniumsalzes mit Silbernitratlösung als weisser Niederschlag erhalten. Das Ammoniumsalz bereitet man durch Einleiten von trocknem Ammoniak in die ätherische Lösung des Esters. Es fällt in langen Fäden aus, die 
sich zu einer bräunlichen, zähen, in Wasser leicht löslichen Masse zusammenballen. Das Silbersalz scheidet sich aus heissem, absolutem Alkohol, in dem es ziemlich schwer löslich ist, beim Erkalten als dicke, weissliche Haut ab.

I. $0,1490 \mathrm{~g}$ gaben $0,0405 \mathrm{Ag}$.

II. $0,3393 \mathrm{~g} \quad, \quad 0,0928 \mathrm{Ag}$. Ber. für $\mathrm{Ag} \quad 27,24$ $\begin{array}{ccc}\mathrm{C}_{15} \mathrm{H}_{14} \mathrm{O}_{5} \mathrm{NAg} & \mathrm{I} & \mathrm{II} \\ 27,24 & 27,18 & 27,37\end{array}$

Das Oel besteht demnach wohl hauptsächlich aus dem AcetphenyJglycoylcyanessigester, dem von den beiden in Betracht kommenden Formeln die Enolformel zukommen dürfte.

Eine merkwürdige Umwandlung erleidet dieser Ester, wenn man ihn mit Methyl- oder Aethylalkohol kocht, oder wenn man ihn mit conc. Salzsäure in Berührung lässt.

Bei dem Versuche, die bei der Einwirkung von Acetylmandelsäurechlorid auf Natriumcyanessigester sich bildenden organischen Natriumsalze vom Kochsalz durch Kochen mit Methyl- oder Aethylalkohol zu trennen, beobachteten wir die Entstehung einer indifferenten Verbindung. Analysen gaben auf die Formel des $\gamma$-Phenyltetron- $\alpha$-carbonsäureäthylesters oder der $\gamma$-Phenyl- $\alpha$ carboxäthyltetronsäure, in der ein Sauerstoffatom durch die Imidogruppe ersetzt ist, stimmende Werthe. Wir halten, wie wir in der Kinleitung darlegten, diese Sub$\operatorname{stanz}$ für

$$
\begin{gathered}
\gamma-\text { Phenyl-c-carboxäthyltetramsäure, } \\
\mathrm{C}_{6} \mathrm{H}_{5} \mathrm{CH}<-\mathrm{CH}(\mathrm{OH})=\mathrm{C} . \mathrm{CO}_{2} \mathrm{C}_{2} \mathrm{H}_{3}
\end{gathered}
$$

oder, da sie keine sauren Eigenschaften mehr besitzt, für die entsprechende Oxoverbindung, das

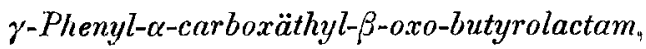

$$
\begin{aligned}
& \mathrm{C}_{8} \mathrm{H}_{5} \mathrm{CH}<\mathrm{NH}-\mathrm{CO}-\mathrm{CH} . \mathrm{CO}_{2} \mathrm{C}_{2} \mathrm{H}_{5}
\end{aligned}
$$

Zur Darstellung dieses Lactams führt man die Reaction von Acetylmandelsäurechlorid auf Natriumcyanessigester, 
wie oben beschrieben, aus, destillirt dann den Aether ab und kocht den Rückstand 2 Stunden unter Rückflusskühlung auf dem Wasserbad mit $100 \mathrm{ccm}$ Methylalkohol oder Aethylalkohol. Während sich das ursprüngliche Reactionsproduct in Wasser löste, gab die vom Kochsalz durch Filtration getrennte methyl- oder äthylalkoholische Lösung beim Vermischen mit Wasser einen weissen, voluminösen, flockigen Niederschlag, der abgesaugt und mehrmals mit heissem Wasser ausgewaschen wurde. Das Filtrat reagirte stark alkalisch und roch nach Essigester. Nach dem Einengen des Filtrates wurde in ihm durch Versetzen mit Salzsäure nur eine geringe Trübung hervorgerufen, ein Zeichen dafür, dass von dem ursprünglichen sauren Ester nichts mehr vorhanden war. Dieselbe Verbindung erhält man auch, wenn man den mit Salzsäure abgeschiedenen ölförmigen Ester mit einem Ueberschuss von rauchender Salzsäure unter häufigem Umschütteln mehrere Tage bei gewöhnlicher Temperatur stehen lässt. Es scheiden sich weisse, dickb]ätterige Krystalle aus.

Die auf einem dieser Wege dargestellte Verbindung ist in Aether, Aceton, Chloroform, Benzol, Xylol und Ligroïn nahezu unlöslich, schwer löst sie sich in kochendem Wasser, aus dem sie sich beim Erkalten als weisses Pulver ausscheidet. Leichter löst sie sich in heissem Alkohol oder Eisessig, aus letzterem Lösungsmittel beim Erkalten in kleinen dickblätterigen Krystallen krystallisirend, die sich über $200^{\circ}$ erhitzt, bräunen und zwischen $220^{\circ}$ und $223^{\circ}$ unter vollständiger Zersetzung schmelzen. Die Analysen gaben auf die Formel $\mathrm{C}_{13} \mathrm{H}_{13} \mathrm{NO}_{4}$ stimmende Werthe. I, II und $\mathrm{V}$ sind mit der durch kochenden AethylalkohoI, III, IV und VI mit der durch rauchende Salzsäure bereiteten Verbindung ausgeführt:
I. $0,1165 \mathrm{~g}$ gaben $0,2710 \mathrm{CO}_{2}$ und $0,0610 \mathrm{H}_{2} \mathrm{O}$.
II. $0,1565 \mathrm{~g} \quad, \quad 0,3640 \quad \mathrm{CO}_{2}, \quad 0,0750 \mathrm{H}_{2} \mathrm{O}$.
III. $0,1930 \mathrm{~g} \quad, \quad 0,4515 \mathrm{CO}_{2}, 0,1010 \mathrm{H}_{2} \mathrm{O}$.
IV. $0,1440 \mathrm{~g} \Longrightarrow \quad 0,3363 \mathrm{CO}_{2}, 0,0733 \mathrm{H}_{2} \mathrm{O}$. 
V. $0,3730 \mathrm{~g} \quad, \quad 19,6 \mathrm{ccm}$ Stickgas bei $23^{\circ}$ und $760 \mathrm{~mm}$ Druck VI. $0,3680 \mathrm{~g} \quad, 18,7 \mathrm{ccm} \quad, \quad 17,5^{\circ}, 753 \mathrm{~mm}$,

\begin{tabular}{cccccccc} 
& Ber. für & \multicolumn{7}{c}{ Gef. } \\
& $\mathrm{C}_{13} \mathrm{H}_{18} \mathrm{NO}_{4}$ & $\mathrm{I}$ & II & III & IV & V & VI \\
$\mathrm{C}$ & 63,13 & 63,44 & 63,44 & 63,80 & 63,69 & - & - \\
$\mathrm{H}$ & 5,30 & 5,77 & 5,36 & 5,85 & 5,69 & - & - \\
$\mathrm{N}$ & 5,68 & - & - & - & - & 5,92 & 5,81 \\
$\mathrm{O}$ & 25,89 & - & - & - & - & - & -
\end{tabular}

Zwei Molekulargewichtsbestimmungen in Phenol gaben auf die einfache Formel stimmende Werthe.

I. $0,4060 \mathrm{~g}$ gaben in $15,953 \mathrm{~g}$ Phenol gelöst, als Schmelzpunktserniedrigung: $1.0,82^{\circ}$, dann nach 7 maliger Wiederholung 2. $0,97^{\circ}$, die letzten drei Bestimmungen gaben denselben Werth.

II. $0,4815 \mathrm{~g}$ wurden der vorigen Lösung hinzugefügt, wodurch die Erniedrigungen: $1.1,79^{\circ}$ bis 2. $1,86^{\circ}$ beobachtet wurden.

Molekulardepression für Phenol $=76$.

Ber. für

Gef.

$$
\begin{aligned}
& \mathrm{C}_{13} \mathrm{H}_{13} \mathrm{O}_{4} \mathrm{~N} \quad \mathrm{I}_{1}, \quad \mathrm{I}_{2} . \quad \mathrm{II}_{1} \text {. } \mathrm{II}_{2} \text {. } \\
& \begin{array}{llllll}
M & 247 & 235,9 & 199,4 & 236,3 & 227,9
\end{array}
\end{aligned}
$$

Das $\gamma$-Phenyl- $\alpha$-carboxäthyl- $\beta$-oxobutyrolactam wird durch Kochen mit Wasser, durch 2-3 stündiges Erhitzen mit trocknem, alkoholischem Ammoniak, durch Auflösen in heissem Anilin oder Phenylhydrazin oder kalter, concentrierter Schwefelsäure und durch Kochen mit Acetylchlorid nicht verändert. Auch löst es sich in kalter, verdünnter Natronlauge nur langsam anf, rascher bei gegelindem Erwärmen. Beim Kochen der alkalischen Lösung entweicht Ammoniak.

Die Einwirkung von Alkalilange auf das Lactam haben wir eingehender untersucht mit der Absicht, das $\gamma$-Phenyl- $\beta$-oxobutyrolactam selbst zu erhalten, aus der zunächst entstehenden $\gamma$-Phenyl- $\beta$-oxobutyrolactam- $\alpha$-carbonsäure. Allein wir stiessen auf unerwartete Schwierigkeiten in so fern, als wir bei der Verseifung des $\gamma$-Phenyl$\beta$-oxo- $\alpha$-carboxäthyl-butyrolactams zwei isomere Körper erhielten, deren Analysen auf die $\gamma$-Phenyl- $\beta$-oxobutyrolactam- $\alpha$-carbonsäure stimmende Werthe ergaben. In 
welchen Beziehungen diese beiden Verbindungen zu dem $\gamma$-Phenyl- $\beta$-ox0- $\alpha$-carboxäthylbutyrolactam stehen, konnten wir bis jetzt nicht aufklären.

$10 \mathrm{~g}$ Ester lösen sich in $20-25 \mathrm{ccm}$ verdünnter Natronlauge beim Erwärmen auf $40-50^{\circ}$ allmählich auf. Nach einer Viertelstunde gab eine Probe mit Salzsäure versetzt einen weissen Niederschlag, der abfiltrirt und ausgewaschen sich in Sodalösung unter Aufbrausen löste. Offenbar war eine starke Säure entstanden. Die gesammte Lösung wurde nun abgekühlt, mit verdünnter Salzsäure gefällt und so die ,Substanz $I^{\text {" }}$ erhalten.

Das Filtrat hinterliess nach dem Eindunsten auf dem Wasserbad einen Trockenrückstand, der mit kochendem Alkohol behandelt sich in zurückbleibendes Kochsalz und in Alkohol lösliche Säuren trennen ließ. Aus der heissen, alkoholisehen Lösung schied sich beim Erkalten „Substanz $I I^{\prime \prime}$ aus die aus Alkohol umkrystallisirt weisse, silberglänzende Schuppen bildet.

Das alkoholische Filtrat von "Substanz II" hinterliess auf dem Wasserbad einen braunen Syrup, der sich auf Zusatz von wenig Wasser leicht in der Wärme löste, beim Erkalten aber einen gelblichweissen, flockigen Niederschlag von sauren Eigenschaften ausfallen liess.

Durch Eindunsten der Mutterlauge dieser Säure entstand wiederum ein Syrup, der mit wenig Wasser versetzt sich in einen weisslichen Brei verwandelte. Um die Säure möglichst vollständig herauszuarbeiten, wurde die breiförmige Masse in der Kälte in wenig Natronlange gelöst und durch Salzsäure gefällt. Abfiltrirt und getrocknet, zeigte die Fällung dieselben Eigenschaften wie die unmittelbar aus demselben Filtrat von "Substanz II“ gewonnene Substanz. Beide Fractionen vereinigt bilden „Sabstanz III", die noch keinen völlig einheitlichen Eindruck machte, so daß wir die damit ausgefíhrten Analysen einstweilen nicht mittheilen werden.

Substanz I löst sich in viel Aethylalkohol beim Kochen auf and fällt beim Abkühlen der Lösung als ein weiches, 
feines, weisses Pulver wieder aus; sie schmilzt bei $178--179^{\circ}$. In Alkalicarbonatlösung löst sie sich unter Entwickelung von Kohlensäure wieder auf. Analysen und Titrationen gaben auf die Formel $\mathrm{C}_{11} \mathrm{H}_{9} \mathrm{O}_{4} \mathrm{~N}$ stimmende Werthe, die also der Carbonsäure des mit Alkalilauge behandelten Fisters entsprechen.

I. $0,1300 \mathrm{~g}$ gaben $0,2865 \mathrm{CO}_{2}$ und $0,0545 \mathrm{H}_{2} \mathrm{O}$.

II. $0,1095 \mathrm{~g} \quad, \quad 0,2425 \mathrm{CO}_{2} \quad, \quad 0,0490 \mathrm{H}_{2} \mathrm{O}$.

III. $0,0905 \mathrm{~g} \quad, \quad 0,2005 \mathrm{CO}_{2} \quad \# \quad 0,0395 \mathrm{H}_{2} \mathrm{O}$.

IV. $0,4210 \mathrm{~g} \quad, \quad 23,8 \mathrm{ccm}$ Stickgas bei $23^{\circ}$ und $759 \mathrm{~mm}$ Druck.

Ber. für

Gef.

$\begin{array}{cccccc} & \mathrm{C}_{11} \mathrm{H}_{8} \mathrm{NO}_{4} & \text { I } & \text { II } & \text { III } & \text { IV } \\ \mathrm{C} & 60,25 & 60,10 & 60,40 & 60,42 & - \\ \mathrm{H} & 4,14 & 4,69 & 5,00 & 4,88 & - \\ \mathrm{N} & 6,40 & - & - & - & 6,36 \\ \mathrm{O} & 29,21 & - & - & - & -\end{array}$

Da die Säure in Wasser schwer löslich ist und auch von Wasser schwer benetzt wird, wurde sie in kochendem Alkohol gelöst, aus dem sie sich bei raschem $\mathrm{Er}^{-}$ kalten als sehr feines Pulver abscheidet. In diese Form gebracht lässt sich die Säure leichter titriren. Die Titrationen wurden mit Phenolphtaleïn als Indicator ausgeführt und etwas übertitrirt.

I. $0,2255 \mathrm{~g}$ brauchten $9,75 \mathrm{ccm}$ Natronlauge (Factor 0,109).

II. $0,1990 \mathrm{~g} \quad, \quad 8,75 \mathrm{ccm} \quad " \quad(\quad, 0,109)$. Ber. für $\mathrm{C}_{11} \mathrm{H}_{9} \mathrm{O}_{4} \mathrm{~N}$

$\mathrm{NaOH} \quad 18,27$

Gef.

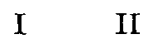

$18,85 \quad 19,09$

Zum Schmelzen erhitzt, spaltet „Substanz I“ Kohlensäure ab. Die braune, beim Erkalten glasartig erstarrende Schmelze löst sich in Alkalilange bei gewöhnlicher Temperatur unter Ammoniakentwickelung.

Substanz II, weisse, silberglänzende Schüppchen, die bei $183^{\circ}$ unter Aufschäumen und Bräunung schmelzen, unterscheidet sich von "Substanz I“ auch durch ihre verschiedene Löslichkeit in Wasser und in Alkohol. Zu unserer Ueberraschung gab sie ebenfalls auf die Formel $\mathrm{C}_{11} \mathrm{H}_{9} \mathrm{NO}_{4}$ stimmende Analysenwerthe, ist also mit der Substanz I isomer. 
I. $0,1735 \mathrm{~g}$ gaben $0,3840 \mathrm{CO}_{2}$ und $0,0680 \mathrm{H}_{2} \mathrm{O}$.

II. $0,1120 \mathrm{~g} \quad, \quad 0,2483 \mathrm{CO}_{2}, 0,0452 \mathrm{H}_{9} \mathrm{O}$.

III. $0,2197 \mathrm{~g} \quad, 13 \mathrm{ccm}$ Stickgas bei $19^{\circ}$ und $757 \mathrm{~mm}$ Druck. Ber. für

Gef. $\mathrm{C}_{11} \mathrm{H}_{9} \mathrm{NO}_{4}$

C 60,25 I II III

H 4,14

$\mathrm{N} \quad 6,40$

O $\quad 29,21$

$0,36 \quad 60,39 \quad-$

$4,38 \quad 4,51 \quad-$

$-\quad-6,78$

$0,1924 \mathrm{~g}$,Substanz II" brauchten $8,15 \mathrm{ccm}$ Natronlauge (Factor 0,109 ).

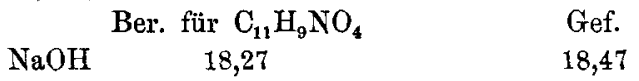

Untersuchungen über die Beziehung dieser beiden offenbar isomeren Substanzen, die wir aus der von uns für $\gamma$-Phenyl-c-carboxäthyl- $\beta$-oxobutyrolactam gehaltenen Verbindung bereiteten, zu einander und zu diesem Lactam sind im hiesigen Institut noch im Gange. 